\title{
A Quantum Inspired Evolutionary Algorithm for Dynamic Multicast Routing with Network Coding
}

\author{
Huanlai Xing ${ }^{1}$ \\ ${ }^{1}$ School of Information Science and Technology \\ Southwest Jiaotong University \\ Chengdu, China \\ hxx@home.swjtu.edu.cn
}

Lexi Xu ${ }^{3}$

${ }^{3}$ Department of Network Optimisation and Management China Unicom Network Technology Research Institute Beijing, China xulx29@chinaunicom.cn

\author{
Rong Qu ${ }^{2}$ \\ ${ }^{2}$ ASAP Group, School of Computer Science \\ University of Nottingham \\ Nottingham, UK \\ rong.qu@nottingham.ac.uk
}

\author{
Zhijian Qu ${ }^{4}$ \\ ${ }^{4}$ College of Computer Science and Technology \\ Shandong University of Technology \\ Zibo, China \\ zhijianqu@gmail.com
}

\begin{abstract}
- this paper studies and models the multicast routing problem with network coding in dynamic network environment, where computational and bandwidth resources are to be jointly optimized. A quantum inspired evolutionary algorithm (QEA) is developed to address the problem above, where a restart scheme is devised for well adapting QEA for tracing the ever-changing optima in dynamic environment. Experimental results show that the proposed QEA outperforms a number of existing evolutionary algorithms in terms of the best solution obtained.
\end{abstract}

Keywords-Dynamic Network Environment; Multicast; Network Coding; Evolutionary Algorithm

\section{INTRODUCTION}

Network coding, as a recent breakthrough communications paradigm, generalizes routing. It allows intermediate nodes within a network to perform mathematical operations to recombine different packets if necessary. Network coding is featured with a number of outstanding advantages, such as natural load balancing, robustness against network failures, stable resistance to attacks, energy saving in multi-hop wireless networks, and so on [1]. Multicast is a technology for point to multi-point data transmission, where the source expects to deliver identical data to a set of receivers [2]. Network coding, when applied to multicast, can always enable the achievement of theoretically maximized throughput [3]. Therefore, network coding based multicast (NCM) has become a hot topic from both academia and industry [1].

NCM data transmission incurs not only link cost but also coding cost (i.e. the computational overhead for packet recombination). In the literature, both costs have been considered. On the one hand, when establishing NCM data transmission, link cost (usage) is expected to be minimized.

This work was supported in part by National Natural Science Foundation of China (No.61401374, No. 61505168), the Project-sponsored by SRF for ROCS, SEM, P. R. China and University of Nottingham, UK.

Huanlai Xing is the corresponding author.
This type of cost comes from flows occupying links, where each link is associated with two non-negative values, i.e. the cost per unit flow and the bandwidth consumption by transmitting that flow. Then the total link cost is the summation of the multiplication of the two values over all links that support NCM. In [4], the minimum-cost multicast routing problem with network coding was modeled as a linear optimization problem. Static and dynamic NCM scenarios were then concerned, where membership of NCM changed over time for dynamic scenarios [4][6].

However, a significant amount of computational and buffering resources is to be consumed due to complicated mathematical functions performed in coding operation(s). A higher coding cost would result into larger packet delivery latencies and slower packet forwarding. A considerable amount of research attention has been dedicated to minimizing the coding cost caused by NCM data transmission, where evolutionary algorithms (EAs) are emphasized. Kim et al. proposed a number of studies in adapting genetic algorithms for minimization of coding operations [7]. In [8], evaluation relaxation techniques were integrated into EAs to reduce the computational overhead. In [9], a population based incremental learning (PBIL) was proposed for tackling the coding cost minimization problem. Besides, a compact genetic algorithm was developed in [10]. It is hence worth studying the problem of jointly optimizing coding and link costs at the same time.

All works above assumed that the network environment was static. However, the nature of real world communications networks is dynamic, where the network environment changes over time and it is difficult to predict changes in advance because there are full of uncertainties in a network. For example, network topology may suddenly vary. Therefore, it is 
necessary to investigate the NCM performance in the dynamic network environment if considering its practical deployment.

Quantum-inspired evolutionary algorithm (QEA) is one of the well-known stochastic meta-heuristics, integrating quantum computation concept into genetic algorithm (GA) [11]. Without explicit population, QEA records the estimated distribution of promising individuals in the search space using quantum-bit representation. By evolving multiple probabilistic models, QEA is able to effectively explore the search space with a very small number of individuals. Hence, it has been widely applied in many fields including network coding related optimization problem [12].

This paper formulates the multicast routing problem with network coding in dynamic network environment, where weighted sum of coding and link costs is to be minimized. Due to the dynamic nature of telecommunications networks, one of the most difficulties in tackling the problem above is optima tracing and localization in ever-changing fitness landscape. In order to tackle the dynamic optimization problem, we propose a novel QEA with a restart scheme embedded. The restart scheme is able to not only detect the network environment change but also guide the search towards unknown yet promising areas in the search space. Performance comparison shows that the proposed QEA outperforms a number of existing evolutionary algorithms (EAs) regarding quality of solutions.

The rest of this paper is organized as follows. Section II formulates the dynamic optimization problem considered in this paper. Section III describes the proposed QEA algorithm in detail. Performance evaluation and comparison is conducted in Section IV. Conclusions are given in Section V.

\section{PROBLEM DESCRIPTION}

This paper models a communications network with dynamic environment at time $t$ as a directed graph $G(t)=\left(V_{t}\right.$, $\left.E_{t}\right) . V_{t}$ is the node set and $E_{t}$ is the link set. For an arbitrary link $e \in E_{t}$, it is associated with a link cost $\varphi_{L}(e)$.

An NCM data transmission scenario is to deliver identical data sent from a source node $s$ to a number of receivers $D=$ $\left\{d_{1}, \ldots, d_{|D|}\right\}$ at an expected data rate $R$, where $|D|$ is the number of receivers, $s, d_{1}, \ldots, d_{|D|} \in V_{t}$ and $R$ is an integer. Given an NCM scenario, the task is to find a connected subgraph in $G(t)$, composed of $R \times|D|$ link-disjoint paths, where each path is from $s$ to one of the receivers, e.g. $d_{k} \in V_{t}$. We call the connected subgraph as NCM subgraph and denote it by $G_{N C M}(t)$ For receiver $d_{k}$, the $i$-th path and its link set at time $t$ are denoted by $P_{i}\left(s, d_{k}, t\right)$ and $\omega_{i}\left(s, d_{k}, t\right)$, respectively. Let $r\left(s, d_{k}, t\right)$ be the achievable data rate between $s$ and $d_{k} \in D$ in $G_{N C M}(t)$.

This paper uses the number of coding links to estimate the amount of coding operations. Suppose there are $M$ coding links, $\varepsilon_{1}, \ldots, \varepsilon_{\mathrm{M}}$, in $G_{N C M}(t)$, where $\varepsilon_{k}, k=1, \ldots, M$, is associated with $\mu_{k}$ incoming data flows to be coded together. As the complexity of coding operation, to a certain extent, depends on the number of data flows being coded, in this paper, the cost per coding operation is proportional to the number of data flows being coded. For coding link $\varepsilon_{k}$, the associated coding cost is $\mu_{k} \cdot \varphi_{C}\left(\varepsilon_{k}\right)$, where $\varphi_{C}\left(\varepsilon_{k}\right)$ is the cost per each flow of $\varepsilon_{k}$.
A list of notations for formulating the dynamic optimization problem is defined as below.

$-s:$ the source node in $G(t)$;

$-D=\left\{d_{1}, \ldots, d_{|D|}\right\}:$ the set of receivers;

$-G_{N C M}(t):$ NCM subgraph at time $t$;

$-P_{i}\left(s, d_{k}, t\right)$ : the $i$-th path of $G_{N C M}(t)$ at time $t$;

- $\omega_{i}\left(s, d_{k}, t\right)$ : the link set of $P_{i}\left(s, d_{k}, t\right)$ at time $t$;

$-r\left(s, d_{k}, t\right)$ : the achievable data rate between $s$ and $d_{k} \in D$ in $G_{N C M}(t)$

$-\varepsilon_{k}$ : the $k$-th coding link in $G_{N C M}(t)$;

$-\mu_{k}$ : the number of data flows to be coded together at $\varepsilon_{k}$;

$-\varphi_{C}\left(\varepsilon_{k}\right)$ : the cost per each flow of $\varepsilon_{k}$.

$-\varphi_{L}(e)$ : the cost per each flow of $e$.

The multicast routing problem with network coding in a dynamic network environment is defined as to find an NCM subgraph, with coding and link costs minimized and data rate constraint satisfied.

Minimize:

$$
\lambda_{1} \cdot\left(\sum_{k=1}^{\mathrm{M}} \mu_{k} \cdot \varphi_{C}\left(\varepsilon_{k}\right)\right)+\lambda_{2} \cdot\left(\sum_{e \dot{\circ} G_{N C M}(t)} \varphi_{L}(e)\right)
$$

Subject to:

$$
\begin{gathered}
r\left(s, d_{k}, t\right)=R, \forall d_{k} \in D \\
\omega_{i}\left(s, d_{k}, t\right) \cap \omega_{j}\left(s, d_{k}, t\right)=\emptyset, \forall d_{k} \in D, \forall i \neq j \in\{1, \ldots, R\}
\end{gathered}
$$

Eq. (1) describes the objective of the problem concerned, where $\lambda_{1}$ and $\lambda_{2}$ are positive real-valued weighted coefficients ( $\lambda_{1}=\lambda_{2}=0.5$ in this paper). Eq. (2) and Eq. (3) are the data rate and link-disjoint paths constraints, respectively.

\section{THE PROPOSED ALGORITHM}

This section first introduces the restart scheme and then describes the proposed QEA.

\section{A. Restart Scheme}

In traditional QEA, Q-bit individuals cannot easily get out of local optima once they converge [11]. However, the problem concerned in this paper is a real-world optimization problem which is based on dynamic network environment. This may bring heavy burdens to the traditional QEA in terms of tracing moving optima in an ever-changing search space. To the best of our knowledge, little consideration has been paid to the above issue. Therefore, this paper presents a restart scheme which helps adapt QEA for the dynamic problem concerned.

In the restart scheme, a small number of samples are maintained to detect environmental change by evaluating their 
fitness values at each generation. More samples lead to more precise detection at the cost of higher computational cost. Let $Q(t)=\left\{q_{1}^{t}, q_{2}{ }^{t}, \ldots, q_{N}{ }^{t}\right\}$ be the population with $N$ Q-bit individuals at generation $t$. Denote the observed population of $Q(t)$ by $P(t)=\left\{\chi_{1}{ }^{t}, \chi_{2}^{t}, \ldots, \chi_{N}{ }^{t}\right\}$. Individual $q_{k}^{t}$ is defined in Eq. (4), where $m$ is the individual length, $\alpha_{i}^{t}$ and $\beta_{i}^{t}$ are complex numbers specifying the probability amplitudes of the corresponding states and $\left|\alpha_{i}^{t}\right|^{2}+\left|\beta_{i}^{t}\right|^{2}=1, i=1, \ldots, m$.

$$
q_{k}^{t}=\left(\begin{array}{cccc}
\alpha_{k 1}^{t} & \alpha_{k 2}^{t} & \ldots & \alpha_{k m}^{t} \\
\beta_{k 1}^{t} & \beta_{k 2}^{t} & \ldots & \beta_{k m}^{t}
\end{array}\right)
$$

Once environmental change happens, the best feasible solution $B=b_{1}, \ldots, b_{m}$ is selected from the observed population and each Q-bit is restarted using Eq. (5), where $\alpha_{1}$ and $\alpha_{2}$ are constant numbers. In this paper, $\left|\alpha_{1}\right|^{2}$ and $\left|\alpha_{2}\right|^{2}$ are set to 0.7 and 0.3 , respectively.

$$
\left(\begin{array}{c}
\alpha_{k i}^{t} \\
\beta_{k i}^{t}
\end{array}\right)= \begin{cases}\left(\sqrt{\left|\alpha_{1}\right|^{2}}, \sqrt{1-\left|\alpha_{1}\right|^{2}}\right)^{T}, & b_{k}=0 \\
\left(\sqrt{\left|\alpha_{2}\right|^{2}}, \sqrt{1-\left|\alpha_{2}\right|^{2}}\right)^{T}, & b_{k}=1\end{cases}
$$

With this restart scheme, the stochastic search is easily guided towards unknown yet promising areas in the search space, which helps quickly adapting to the new environment.

\section{B. The Proposed QEA}

Binary link state (BLS) encoding is used as individual representation. For an individual $\chi_{k}{ }^{t}$, its fitness value is set to be a sufficiently large number (i.e. 1200 in this paper) if $\chi_{k}^{t}$ is feasible; Otherwise, the fitness value is set based on Eq. (1). The overall procedure of the proposed QEA is illustrated in Fig. 1.

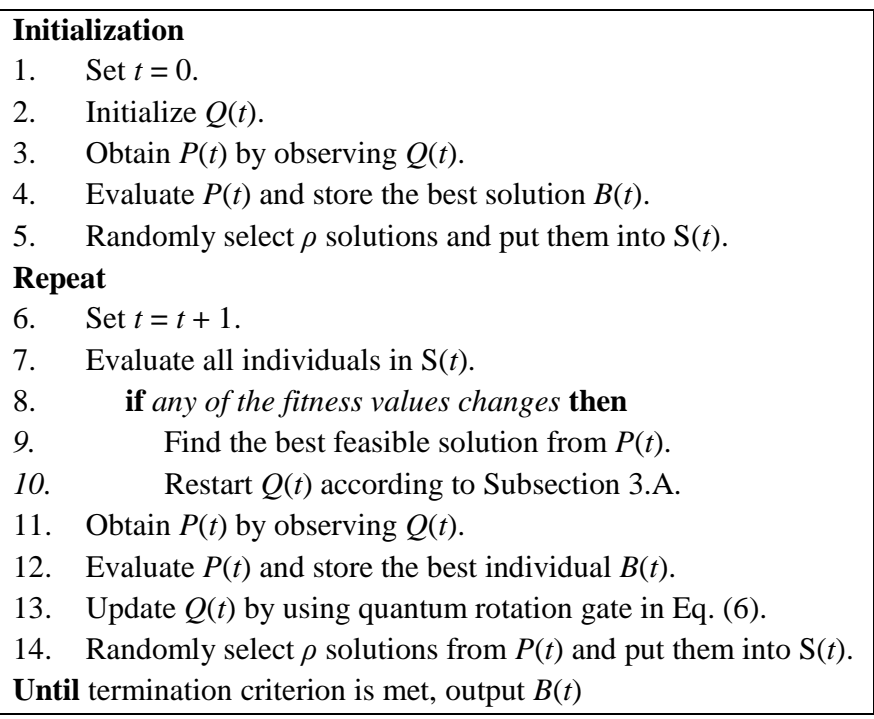

Fig. 1. Overall procedure of the proposed QEA
In Step 13, the $i$-th Q-bit value $\left(\alpha_{k i}^{t}, \beta_{k i}{ }^{t}\right)^{T}$ of $q_{k}^{t}$ is updated in Eq. (6), where $U(t)$ is the quantum rotation gate. Details can be found in [11][12]. The search stops once a predefined number of generations are evolved.

$$
\left(\begin{array}{l}
\alpha_{k i}^{t} \\
\beta_{k i}^{t}
\end{array}\right)=U(t)\left(\begin{array}{c}
\alpha_{k i}^{t-1} \\
\beta_{k i}^{t-1}
\end{array}\right)
$$

\section{PERFORMANCE COMPARISON}

To evaluate the performance of the proposed QEA, we compare a number of existing evolutionary algorithms (EAs), as listed below.

- tGA: traditional GA [7].

- rGA: tGA with a restart scheme, where the restart scheme re-initializes the population once the environment is changed.

- tQEA: QEA for minimizing network coding resources [12].

- rQEA: the proposed QEA.

Ten test instances are used for performance comparison, including 3-copy, 7-copy and 8 random networks. Random networks are Rd-1 (20nodes, 37links), Rd-2 (20nodes, 39links), Rd-3 (30nodes, 60links), Rd-4 (30nodes, 69links), Rd-5 (40nodes, 78links), Rd-6 (40nodes, 85links), Rd-7 (50nodes, 101links), and Rd-8 (50nodes, 118links). Link cost $\varphi_{L}(e)$ for link $e$ is distributed uniformly in the range of $[5,15]$. Coding cost per each data flow $\varphi_{C}$ is set to 10 .

The dynamic problem generator presented in [13] is utilized to generate all dynamic problems, where the environment changes every 50 generations and there are three changes incurred in each test instance. The population size and the predefined number of generations are set to 20 and 200, respectively. All experiments were implemented on a Windows XP computer with Intel Core(TM) E8400 3.0GHz, 2G RAM. Each algorithm for comparison is run 20 times on each instance.

To measure performance of each algorithm, for each run, we average the best fitness values obtained from each stationary period. The average best fitness $(\mathrm{ABF})$ value of each algorithm is able to reflect the overall performance of each algorithm. The mean value and the standard deviation are collected in Table 1.

Table 1 clearly shows that the two QEAs gain the best performance than GAs, reflecting the superiority of QEAs in solving the dynamic optimization problem concerned in the paper. Table 1 also shows that rQEA outperforms tQEA in all instances. It is too hard for tQEA to repeatedly initiate the exploration over vast areas in the search space once the environment changes, which leads to serious performance deterioration in terms of global exploration. 


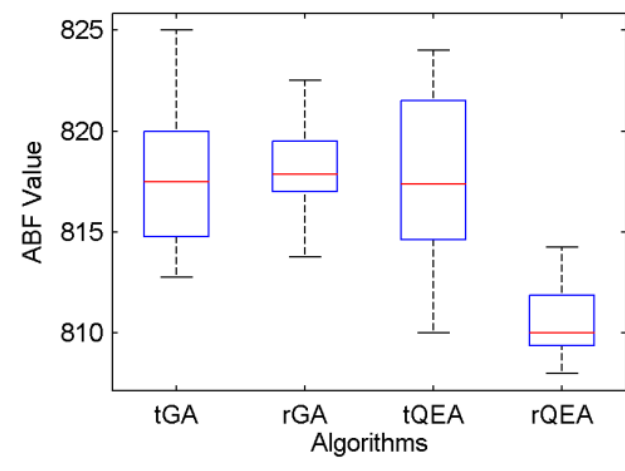

(a) 7 copy

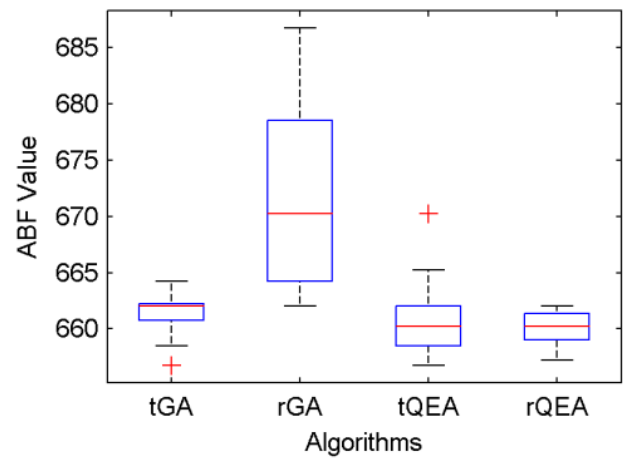

(c) RD-6

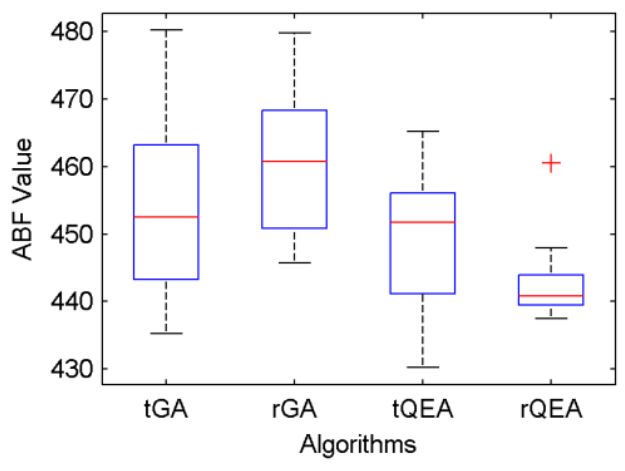

(b) RD-4

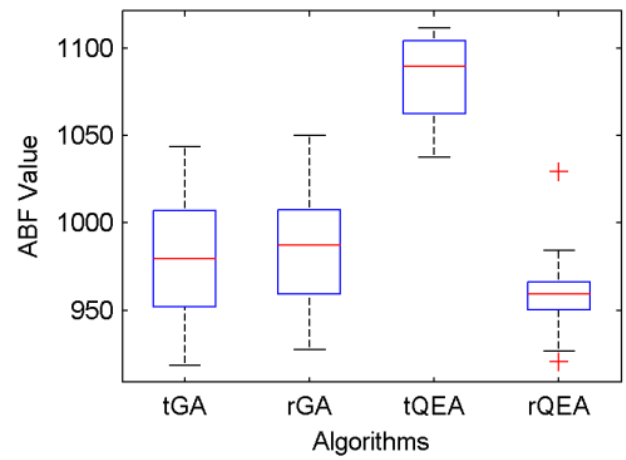

(d) RD-8

Fig. 2. Box plots of tGA, rGA, tQEA and rQEA on four selected instances.

TABLE I. RESUlTS OF MEAN VALUE AND STANDARD DEVIATION (BEST RESUlts ARE IN BOLD)

\begin{tabular}{lcccc}
\hline Instances & tGA & rGA & tQEA & rQEA \\
\hline 3copy & $365.2(0.5)$ & $365.1(0.5)$ & $364.1(0.3)$ & $\mathbf{3 6 4 . 0}(0.0)$ \\
7copy & $817.8(3.5)$ & $818.0(2.3)$ & $817.6(4.0)$ & $\mathbf{8 1 0 . 6}(1.6)$ \\
Rd-1 & $363.9(5.8)$ & $384.7(18.2)$ & $359.5(9.4)$ & $\mathbf{3 5 8 . 6}(5.6)$ \\
Rd-2 & $295.0(9.7)$ & $303.4(14.4)$ & $285.5(6.5)$ & $\mathbf{2 8 4 . 1}(4.8)$ \\
Rd-3 & $447.4(9.5)$ & $464.7(23.7)$ & $439.7(9.7)$ & $\mathbf{4 3 7 . 8}(5.0)$ \\
Rd-4 & $453.3(12.1)$ & $460.6(11.0)$ & $449.3(10.5)$ & $\mathbf{4 4 2 . 4}(5.1)$ \\
Rd-5 & $637.7(27.7)$ & $670.6(33.8)$ & $628.8(16.4)$ & $\mathbf{6 1 8 . 6}(10.6)$ \\
Rd-6 & $661.4(1.9)$ & $671.6(7.9)$ & $660.5(3.1)$ & $\mathbf{6 6 0 . 1}(1.4)$ \\
Rd-7 & $650.2(20.4)$ & $689.2(44.1)$ & $684.5(20.5)$ & $\mathbf{6 2 8 . 8}(17.7)$ \\
Rd-8 & $980.8(35.1)$ & $988.4(35.6)$ & $1083.9(22.7)$ & $\mathbf{9 6 0 . 0}(22.3)$ \\
\hline
\end{tabular}

With the restart scheme integrated, rQEA can not only detect the environmental change but also follow the trajectory of moving optima in a dynamic environment. This is why rQEA is always able to adapt to the problem concerned in the paper.

Fig. 2 illustrates the box plots of the four algorithms on four selected instances, respectively, including 7copy, Rd-4, Rd-6 and Rd-8. Apparently, rQEA outperforms tGA, rGA and tQEA, regarding the $\mathrm{ABF}$ value distribution. rQEA obtains the best $\mathrm{ABF}$ distribution in all instances. It achieves the best maximum, Q3, median, Q1, and minimum values in the box plot. Hence, Fig.2 is also able to demonstrate the superiority of rQEA over tGA, rGA, and tQEA.
Fig. 3 shows the curve of ABF value of each algorithm on the same instances above. One can easily find that rQEA (by solid black line) obtains the best $\mathrm{ABF}$ value after most of environmental changes. For instance, rQEA outperforms the other algorithms after each environmental change(see Fig.3(b)) Besides, QEAs outperform GAs in most of the instances. It is because of the nature of QEA. With the estimated distribution of promising samples maintained, QEA evolves a set of probabilistic stochastic models in parallel, helping guide the search towards the exploration of dynamic and unpredictable search space efficiently. On the other hand, GA is less competitive due to that explicit population is less adaptive to the environmental change.

\section{CONCLUSIONS}

This paper formulates a dynamic multicast routing problem with network coding, where minimizing weighted sum of coding and link costs is the single objective. It develops a quantum-inspired evolutionary algorithm (QEA), where a restart scheme is put forward for helping the algorithm always tracing promising regions where optima reside. Experimental results illustrate that the proposed QEA performs better than a number of existing evolutionary algorithms regarding the best solution obtained. The underlying model for the optimization problem in the paper is based on wired network. It is quite possible to bring ample benefits to wireless communications. The adaptation of the proposed algorithm for multicast with network coding in wireless communications system, e.g. LTE cellular systems [14][15], will be considered in the future. 


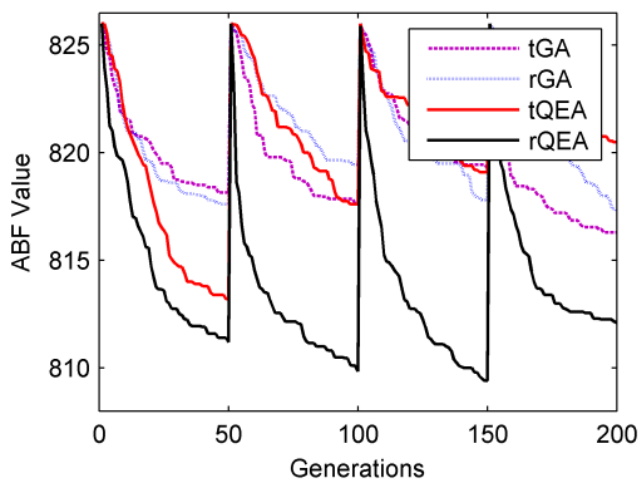

(a) 7 copy

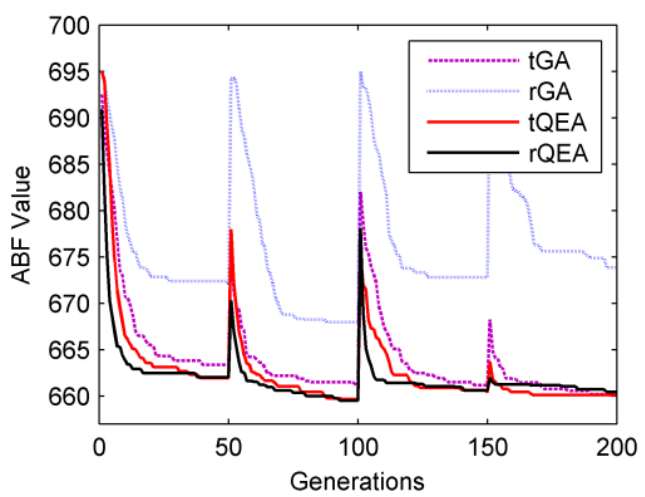

(c) RD-6

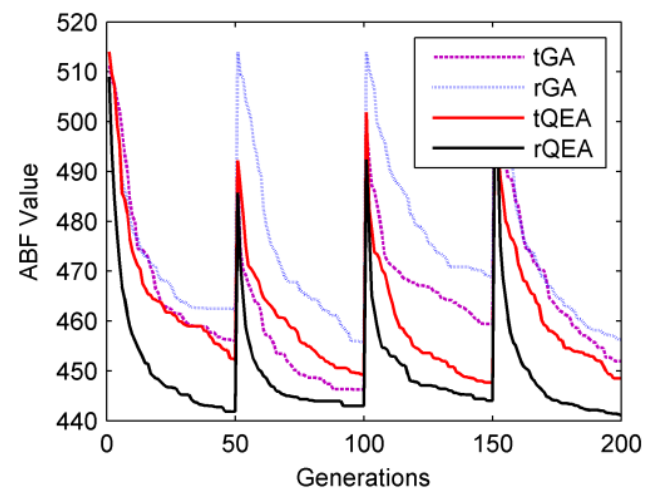

(b) RD-4

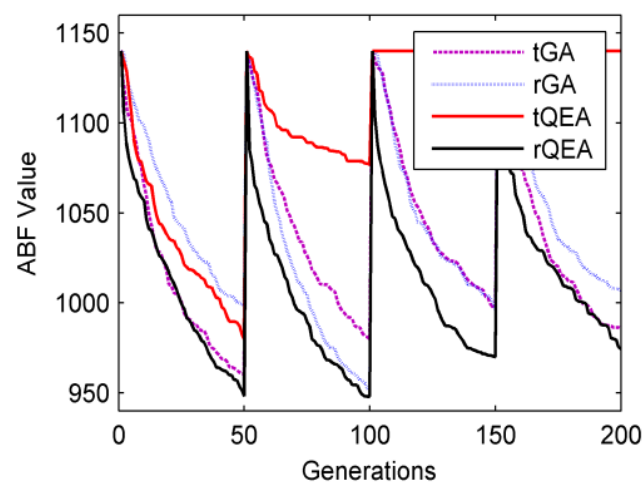

(d) RD-8

Fig. 3. ABF value curves on four selected instances.

\section{REFERENCES}

[1] R. Bassoli, H. Marques, J. Rodriguez, K.W. Shum, and R. Tafazolli, "Network coding theory: a survey," IEEE Commun. Surv. \& Tutor., vol.15, pp. 1950-1978, 2013.

[2] A. Benslimane, Multimedia Multicast on The Internet, Wiley: ISTE, 2007.

[3] S.Y.R. Li, R.W. Yeung, and N. Cai, "Linear network coding," IEEE Trans. Info. Theory, vol.49, pp. 371-381, 2003.

[4] D.S. Lun, M. Médard, T. Ho, and R. Koetter, "Network coding with a cost criterion," in Proc. ISITA2004, Parma, Italy, 2004.

[5] S. Bhadra, S. Shakkottai, and P. Gupta, "Min-cost selfish multicast with network coding," IEEE Trans. Info. Theory, vol.52, pp. 5077-5087, 2006.

[6] F. Zhao, M. Médard, D.S. Lun, and A. Ozdaglar, "Minimum cost subgraph algorithms for static and dynamic multicasts with network coding," In: Tarokh V (ed.) New Directions in Wireless Communications Research, Springer, pp.317-349, 2009.

[7] M. Kim, M. Médard, V. Aggarwal, V.O. Reilly, W. Kim, C.W. Ahn, and M. Effros, "Evolutionary approaches to minimizing network coding resources,” In Proc. INFOCOM2007, pp.1991-1999, 2007.

[8] C.W. Ahn, "Fast and adaptive evolutionary algorithm for minimum-cost multicast with network coding," Electronics Letters, vol. 47, no.12, pp. 700-701.
[9] H. Xing and R. Qu, "A population based incremental learning for network coding resources minimization," IEEE Comm. Lett. Vol.15, 698-700, 2011

[10] H. Xing and R. Qu, "A compact genetic algorithm for the network coding based resource minimization problem," App. Intell., vol.36, no.4, pp. 809-823, 2012.

[11] G. Zhang, "Quantum-inspired evolutionary algorithm: a survey and empirical study," J. Heuristics, vol.17, 303-351, 2011.

[12] H. Xing, Y. Ji, L. Bai, and Y. Sun, “An improved quantum-inspired evolutionary algorithm for coding resource optimization based network coding multicast scheme," AEU - Int. J. of Electron. and Commun., vol.64, pp.1105-1113, 2010.

[13] S. Yang and X. Yao, "Population based incremental learning with associated memory for dynamic environments," IEEE Trans. Evolut. Comput., vol.12, pp. 542-561, 2008.

[14] L. Xu, Y. Chen, K.K. Chai, J. Schormans, L. Cuthbert, "Self-organising cluster-based cooperative load balancing in OFDMA cellular networks," Wily Wireless Commun. Mob. Comput., vol.15, pp.1171-1187, 2015.

[15] L. Xu, X. Cheng, Y. Chen, K. Chao, D. Liu, H. Xing, "Self-optimised coordinated traffic shifting scheme for LTE cellular systems," In Proc. ICSON2015, Beijing, P.R.China, 2015. 